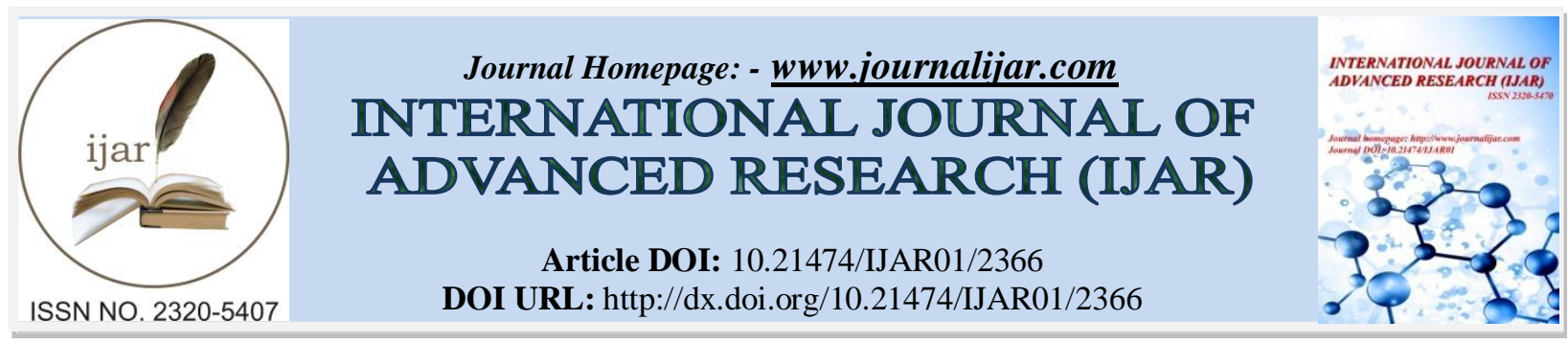

RESEARCH ARTICLE

\title{
A SURVEY ON FACE RECOGNITION SYSTEM AND ITS TECHNIQUES.
}

\author{
Raveendra $\mathbf{K}^{1}$ and Ravi $\mathbf{J}^{\mathbf{2}}$. \\ 1. Government Engineering College, K R Pet, Karnataka, India. \\ 2. Global Academy of Engineering, Bangalore, Karnataka, India.
}

\section{Manuscript Info}

Manuscript History

Received: 18 October 2016

Final Accepted: 20 November 2016

Published: December 2016

Key words:-

Eigen faces Fisher Fisher faces, neural network and Pattern recognition.

\section{Abstract}

The information and data gathering in plenitude, there is an essential requirement for high security. Biometrics has now gotten more consideration. Face biometrics, a valuable tool for a man's verification is a basic and non-meddlesome technique that perceives face in complex multidimensional visual model and builds up a computational model for it. This paper reveals the recognition of the face and then followed by examining the strategy and working. From the above observations, we focused our attention to the latest face recognition techniques posting their favourable circumstances and inconveniences. A few procedures determined here were additionally enhance the proficiency of face recognition under different brightening and demeanor state of face images. The goal of this paper is to present a critical survey of existing literatures on human face recognition.

Copy Right, IJAR, 2016,. All rights reserved.

\section{Introduction:-}

Face recognition gets to be a standout amongst the most biometrics confirmation strategies from the previous couple of years. Face recognition is an intriguing and fruitful use of Pattern recognition and Image investigation. Face recognition is one of the primary biometric technologies and one of the active research fields that recognize a person using facial characteristics of human beings [22]. Face recognition framework has two primary errands: confirmation and distinguishing proof. Face confirmation implies a 1:1 match that thinks about a face images against a layout face images whose personality being guaranteed. Face recognizable proof means a 1: $\mathrm{N}$ issue that looks at a question face image against all image formats in a face database. Machine recognition of appearances is continuously turning out to be imperative because of its extensive variety of business and law implementation applications, which incorporate scientific recognizable proof, get to control, fringe observation and human associations and accessibility of minimal effort recording gadgets. Different biometric components can be utilized with the end goal of human recognition like unique mark, palm print, hand geometry, iris, face, discourse, walks, signature and so forth. The issues with unique finger impression, iris palm print, discourse, strides are they require dynamic co-operation of individual while face recognition is a procedure does not require dynamic co-operation of a man so without teaching the individual can perceive the individual. So face recognition is a great deal more invaluable contrasted with alternate biometrics. Face recognition has a high recognizable proof or recognition rate of more noteworthy than $90 \%$ for colossal face databases with all around controlled stance and brightening conditions.

As a standout amongst the best utilizations of example recognition, image analysis and comprehension, face recognition of late gotten noteworthy consideration, particularly amid the previous quite a long while. This is 
confirm by the rise of face recognition gatherings, for example, AFGR, AVBPA, CV, PR, CVPR, WACV, CGIPV, WCVBVSMA, CCVHM, SSWNN, IP, and efficient observational assessments of Face Recognition Innovation (FRT), including the FERET and XM2VTS conventions. There are no less than two explanations behind this pattern: the first is the extensive variety of business and law requirement applications, and the second is the accessibility of possible advancements following 30 years of research. Facial recognition is a very common application of machine vision. There are many approaches to facial recognition, including both holistic and local comparisons [17].

The past written works on orderly experimental assessments of face recognition are essentially the prior studies by Samal and Iyengar 92on non-connectionist approaches, by Valentin et al. in 94 on connectionist conspires, the copious and careful review by Chellappa et al. in 1995 on 20 years of face recognition, the more drawn out and more extensive reviews by Fromherz in 1997, and by Zhou et al., in 2000. They concentrated on the improvement of face recognition before the mid 1997. Amid the previous quite a long while, face recognition has gotten expanded consideration and has progressed in fact. Numerous business frameworks utilizing face recognition are currently accessible. Noteworthy research efforts have been centered around video-based face demonstrating, handling and recognition.Gender-targeted surveillance can assist with monitoring gender-restricted areas and elevated threat levels that might be associated with a specific gender [19][20][21]. In research work, we empirically evaluate face recognition which considers both shape and texture information to represent face images based on Local

Binary Patterns for person-independent face recognition.[15][16]

\section{Face Recognition System:-}

There are fundamentally three methodologies for face recognition [1]:

\section{Include base approach:-}

In highlight based approach the neighborhood highlights like nose, eyes are fragmented and it can be utilized as info information as a part of face identification to less demanding the assignment of face recognition.

\section{Holistic approach:-}

In all encompassing methodology the entire face taken as the contribution to the face discovery framework to perform face recognition.

\section{Half breed approach:-}

Half breed approach is mix of highlight based and all encompassing methodology. In this approach both neighborhood and entire face is utilized as the contribution to face discovery, programmed face recognition frameworks are included three stages. Their essential flow diagram is given in Figure 1. Among them, identification may incorporate face edge discovery, division and confinement, specifically getting a pre-handled power face image from an information scene, either straightforward or messed, finding its position and dividing the image out of the foundation. Highlight extraction may mean the securing of the image highlights from the image, for example, visual components, factual pixel framework.

Highlights, change coefficient highlights, and arithmetical elements, with accentuation on the logarithmic elements, which speak to the inborn traits of an image.

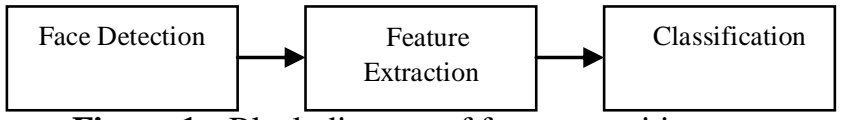

Figure 1:- Block diagram of face recognition

In the Face recognition, the information image is additionally called as test and the database is called as exhibition. At that point it gives a match report and afterward the grouping is done to distinguish the sub-populace to which new perceptions have a place [2].

\section{Face Recognition techniques:-}

\section{Eigen faces:-}

The Eigen face technique is one of the by and large utilized calculations for face recognition. Karhunen-Loeve depends on the Eigen faces system in which the Principal Component Analysis (PCA) is utilized. This technique is effectively used to perform dimensionality lessening. Key Component Analysis is utilized by face recognition and 
location. Numerically, Eigen faces are the vital parts separate the face into highlight vectors. The element vector data can be acquired from covariance framework. These Eigenvectors are utilized to measure the variety between various countenances. The appearances are described by the straight blend of most noteworthy Eigen values. Every face can be considered as a straight blend of the Eigen faces. The face can be approximated by utilizing the eigenvectors having the biggest Eigen values. The best $\mathrm{M}$ Eigen faces characterize an $\mathrm{M}$ dimensional space, which is called as the "face space". Important Component Analysis is likewise utilized by L. Sirovich and M. Kirby to proficiently speak to images of appearances. They characterized that a face images could be roughly remade utilizing a little gathering of weights for every face and a standard face image. The weights portraying every face are acquired by anticipating the face image onto the eigen image [3].

Eigen face is a viable approach for face recognition. As a result of the straightforwardness of its calculation, execution of an eigen face recognition framework turns out to be simple. It is effective in preparing time and capacity. PCA diminishes the measurement size of a image in a brief timeframe. There is a high relationship between the preparation information and the recognition information. The exactness of eigen face relies on numerous things. As it takes the pixel esteem as examination for the projection, the exactness would diminish with shifting light power. Preprocessing of image is required to accomplish attractive result. Favorable position of this calculation is that the Eigen faces were imagined precisely for those reasons what makes the framework exceptionally productive [7].

A downside is that it is touchy for helping conditions and the position of the head. Impediments Finding the eigenvectors and eigen values are tedious on Probability Prototype Chromosome(PPC). The size and area of every face image must stay comparable PCA (Eigen face) approach maps elements to guideline subspaces that contain generally energy.

\section{Fisher faces:-}

The fisher faces technique utilizes the benefit of inside class data so it minimizes the variety inside class, so the issue with varieties in similar images, for example, lighting varieties can be overcome [2].

A Fisher face is one the most effectively generally utilized technique for face recognition. It depends on appearance technique. In 1930 R.A Fisher created straight/fisher Discriminant analysis for face recognition.[10] It demonstrates effective result in the face recognition prepare.[11] All utilized LDA to discover set of premise images which augments the proportion of between-class dissipate to inside class scramble. The impediment of LDA is that inside the class the disperse framework is constantly single, since the quantity of pixels in images is bigger than the quantity of images so it can build discovery of blunder rate if there is a variety in posture and lighting condition inside same images. So to conquer this issue numerous calculations has been proposed.

The fisher face strategy for face recognition depicted by Belhumeur et al [11] utilizes both primary segment examination and straight discriminant investigation which create a subspace projection framework, comparative as utilized as a part of the eigen face technique. Be that as it may, the fisher face technique can exploit inside class data, minimizing variety inside every class, expanding class partition. Like the eigen face development prepare, the initial step of the fisher face technique is take each $(N x M)$ image exhibit and reshape into a $\left(\left(N^{*} M\right) \times 1\right)$ vector.

Fisher face is like Eigen face however with improvement of better characterization of various classes image. With FLD, one can arrange the preparation set to manage diverse individuals and distinctive outward appearance. We preferred precision in outward appearance over Eigen face approach. Plus, Fisher face evacuates the initial three important segments which are in charge of light force transforms; it is more invariant to light power. [7]

The weaknesses of Fisher face are that it is more mind boggling than Eigen face to finding the projection of face space. Figuring of proportion of between-class dissipate to inside class diffuse requires a considerable measure of preparing time. Moreover, because of the need of better arrangement, the measurement of projection in face space is not as conservative as Eigen face, results in bigger stockpiling of the face and additionally handling time in recognition. [7]

Neural Network:-

The regionally connected neural network examined small windows of an image and decided whether each window contained a face. The system arbitrated between multiple networks to improve performance over a single network. 
In hybrid neural method isto locate human eyes. In the new neural network model proposed, the Constrained Generative Model (CGM), per-formed an accurate estimation of the face set, using a small set of counter-examples. The neural network layers in [8] were shown in Figure 2. Neural networks have been applied, with considerable success, to the problem of frontal face detection. A neural network based upright frontal face detection system was presented in [9].The use of three layers of weights allows evaluating the distance between an input image and the set of face image.

The neural systems are utilized as a part of numerous applications like face recognition issues, character recognition, question recognition and self-governing robot driving. The fundamental goal of the neural system in the face recognition is the attainability of preparing a framework to catch the mind boggling class of face examples. To get the best execution by the neural system, it must be broadly tuned number of layers, number of hubs, learning rates, and so on. The neural systems are non direct in the system so it is broadly utilized strategy for face recognition. Along these lines, the element extraction step might be more effective than the Principal Component Analysis. The creators accomplished $96.2 \%$ exactness in the face recognition handle when 400 images of 40 people. The characterization time is under 0.5 seconds, however the preparation time is the length of 4 hours highlights in a progressive arrangement of layers and gives halfway invariance to interpretation, scale, and miss happening. The disservice of the neural system approach is that when the quantity of classes increments. Edge tracking algorithm is used to obtain the sub windows from the enhanced image. Edges and features are obtained and these feature values are passed into a trained back propagation neural network to sort the sub-window as either face or non-face. The result of the proposed method gives better performance in terms of processing time in testing and training [22].

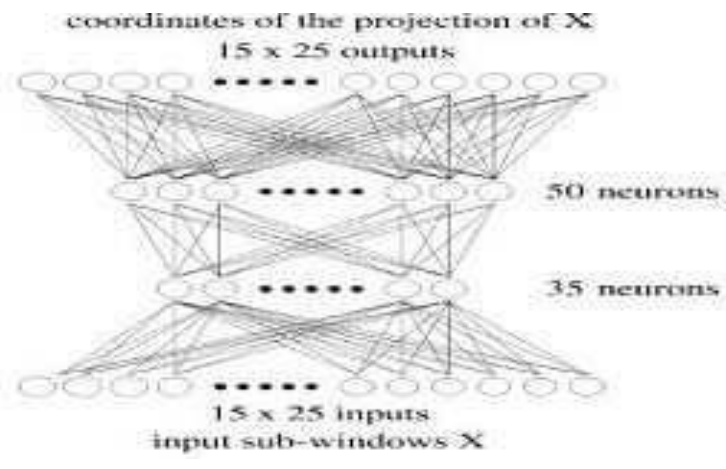

Fig. 2:- Neural network layers.

\section{Spatial Matching Detector Technique:-}

This approach embraces the Support Vector Machines, various template matching methods, other discriminable Kernel Cost Function methods, and so on. It offered a novel detection method, which worked well even in the case of a complicated image collection of detected images which was called a multi-template. Only images which passed the threshold test imposed by the first detector were examined by the second detector, etc. The algorithm's performance compared favorably to the well-known Eigen face and support vector machine based algorithms, but was substantially faster. A schematic description of the geometry behind anti-face algorithm was presented in Figure 3. The algorithm's "positive set" (the images it classifies as members of the multi-template), is orthogonal to the direction around which random images cluster, hence, there are relatively few false alarms.

Schematic Description of the Detection

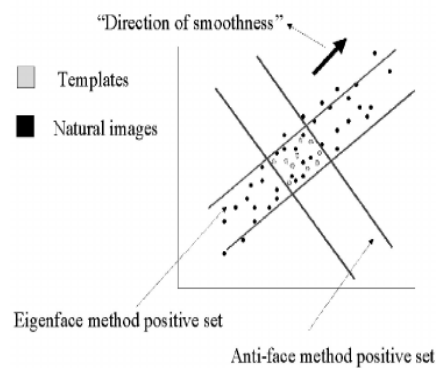

Fig. 3:- Schematic description of the anti-face algorithm 


\section{Fuzzy Theory Based Method:-}

This approach identifies face $\mathrm{s}$ in shading images in view of the fluffy hypothesis. Face Detection from fuzzy theory based method is a run of the mill case of fluffy recognition class. In this paper Wu et al made two fluffy models to depict the skin and hair shading, in which utilized a perceptually uniform shading space to portray the shading data to build the exactness and stableness. Moreover, the models were utilized to separate the skin and hair shading districts, and then contrasting them and the pre-assembled head-shape models by utilizing a fluffy hypothesis based example coordinating strategy to distinguish face hopefuls.

\section{Template matching Technique:-}

In format coordinating, we can misuse other face layouts from various prospects to describe single face. Essentially, dark levels that match the face image can likewise be prepared in appropriate arrangement. In Bruneli and Poggio (1993) the Pop and Bruneli is accessible for all parts of creating programmed four format highlights i.e., eyes, nose, mouth, face and selecting the whole set. The framework is assessed by contrasting results from geometrical construct calculations with respect to 188 images of 47 subjects. The example coordinating calculation is an exceptionally pragmatic approach, extremely easy to utilize and roughly accomplishes better recognition rate. The Principal Component Analysis utilizing Eigen face gives the direct plan of formats. The primary favorable position of this approach is that it is anything but difficult to actualize and is less costly than whatever other component classifier. Relatively, format based calculations are more costly and can't be effectively handled. Be that as it may, the recognition procedure is effectively taken care of between the given format and info image. The many-sided quality emerges just amid the extraction of format. For the most part format based procedures best when contrasted with highlight based strategies. Karungaruet al. (2004) utilizes layout based hereditary calculation and uncovered diverse results on target image by changing the extent of the format as preprocessing. The edge identification and YIQ shading layouts are misused. The outcomes are taken around the separation measure face recognition approach and correlation is performed with existing techniques. Anlonget al. (2005) the creator takes a shot at the framework to build solid and legitimate base. This strategy is exceedingly powerful for bigger databases that take care of the issue of face recognition under sensible computational cost. Sao and Yegnanarayana (2007) a calculation is proposed for individual confirmation utilizing layout based face recognition strategy. Essentially, the tenseness based face representation is figured to process one dimensional images.

Dynamic Time Warping (DTW) and Long Short Term Memory (LSTM) are researched under the Neural Network grouping in which a solitary element layout is sufficiently expansive for highlight extraction. It really executes the angle based learning calculation by taking care of related inclination issues. The trial result uncovers that both techniques perform well for face recognition while the learning procedure gives powerful recognition rate. The working of this approach is summed up by saying that further upgrades are still required keeping in mind the end goal to take care of the recognition issue that is by all accounts extremely basic in true. [4]

A basic rendition of layout coordinating is that a test image spoke to as a two-dimensional exhibit of force qualities is looked at utilizing a reasonable metric, for example, the Euclidean separation, with a solitary format speaking to the entire face. There are a few other more refined adaptations of layout coordinating on face recognition. One can utilize more than one face format from various perspectives to speak to an individual's face. A face from a solitary perspective can likewise be spoken to by an arrangement of numerous unmistakable littler formats. The face image of dark levels may likewise be legitimately prepared before coordinating. Since the primary segments (otherwise called eigenfaces or eigen features) are direct mixes of the layouts in the information premise, the strategy can't accomplish preferable results over relationship, yet it might be less computationally costly .One downside of format coordinating is its computational intricacy. Another issue lies in the portrayal of these layouts. Since the recognition framework must be tolerant to specific disparities between the format and the test image, this resilience may normal out the distinctions that make singular face s novel. By and large, format based methodologies contrasted with highlight coordinating are a more legitimate approach. In synopsis, no current method is free from restrictions. Facilitate endeavors are required to enhance the exhibitions of face recognition strategies, particularly in the extensive variety of situations experienced in certifiable. [5][6]

\section{Curvlet with PCA:-}

The curvelet with PCA distinguishes a component space to address the issue of human face recognition from the database images. The face recognition framework depends on Principal Component Analysis. By utilizing PCA the components can be extricated. The multi determination Curvelet change can be utilized for the productive face image recovery [12]. At the point when contrasted with wavelet has better directional and edge representation. The 
face images can be deteriorated while applying the Curvelet change and the curvelet sub groups can be shape. Likewise the PCA can be utilized for dimensionality lessening. At that point the PCA can apply for each curvelet sub groups and make highlight set. Mahalanobis distance measure can be utilized to quantify the separation between the inquiry and the database images. The notable face database demonstrates the capability of this curvelet based component extraction and gives great recovery result. The exploratory results demonstrate our approach is essentially superior to the customary techniques [12].

\section{DWT and Feature Vector Compression:-}

Face Recognition is critical Biometric certifications for distinguishing proof or check of a person. This will propose a novel method of creating compacted one of kind elements of face images which helps in enhancing coordinating rate of acknowledgment [18]. The preparation face database tests are connected to 2D-DWT to acquire LL band highlights. The LL band elements are subjected to standardization to scale the greatness values in the range 0 to 1 . The yield of standardization is further convolved with the first face test to get remarkable elements. The convolved yield is subjected to Gaussian filter to get smoothened image highlights. Advance, The component vector of a few image tests of single individual are compacted to change over into single vector to database include vectors are made by packing highlight vectors of single individual face tests into single segment extraordinary vectors which helps in downsizing of highlight vectors and enhance coordinating speed. The tests are subjected to same procedure to create special packed test include vectors and are contrasted and database vectors utilizing Euclidean separation. The outcomes are arranged for various arrangement of face databases Furthermore contrasted with existing strategies which approves the execution of proposed strategy [18].The DT-CWT is an effective approach for implementing a wavelet transforms. This technique has been used to incorporate the good properties of Fourier Transformation in the Wavelet Transformation [22].

\section{Other approaches:-}

Beside the above-mentioned approach to face recognition, some researchers also used other methods to perform the studies on face recognition, i.e., the rules of the shape and albedo of a face under all possible illumination conditions, Bayesian decision, etc. Georghiades et al. 2001 [2] presented a generative appearance-based method for recognition human face under variation in lighting and viewpoint, exploiting the fact that the set of images of an object in fixed pose, but under all possible illumination conditions, is a convex cone in the space of images. In [3] Moghaddam et al. 2000 utilized Bayesian decision for the purpose of face recognition and image retrieval. They arrived at the conclusion that Bayesian method gained better results than standard Eigen face method and effectively halved the error rate of eigen face matching. Figure 4 in [3] highlighted the performance difference between standard eigen faces and the Bayesian method from a small test set of $800+$ individuals. Yet other methods have been proposed for 3D face recognition based on local features, local and global geometric cues [13], profiles, and the rank-based decision fusion of various shape-based classifiers [14].

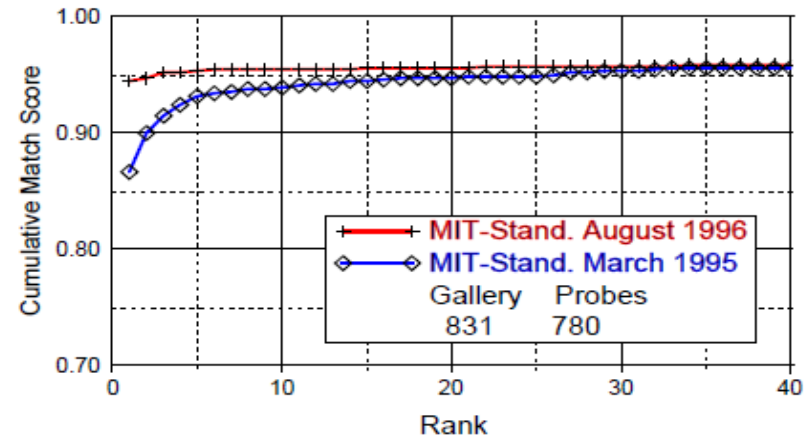

(a) 


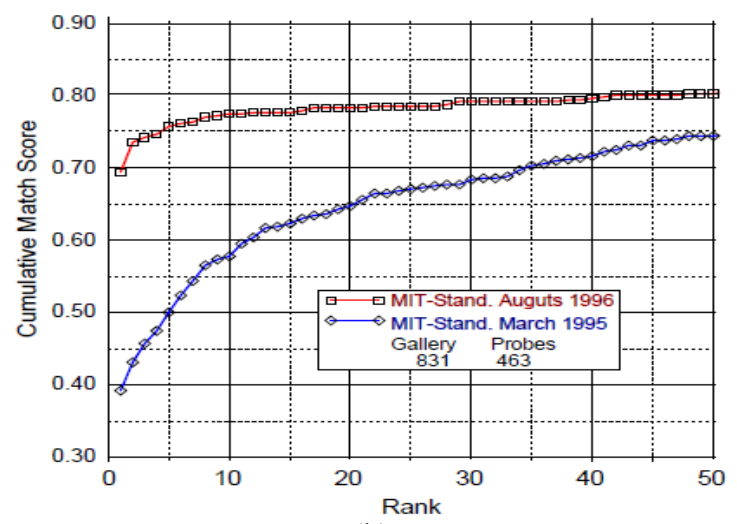

(b)

Figure 4:- Cumulative recognition rates for with standard eigen face matching and the newer Bayesian similarity metric (a) frontal FA/FB views, (b) frontal duplicate views.

Figure 4(a) shows the performance comparison between standard eigen face and the Bayesian method from this test. Note the $10 \%$ gain in performance afforded by the new Bayesian similarity measure. Similarly, Figure 4(b) shows the recognition results for "duplicate" images which were separated in time by up to 6 months (a much more challenging recognition problem) which shows a 30\% improvement in recognition rate with Bayesian matching. Thus we note that in both cases (FA/FB and duplicates) the new probabilistic similarity measure has effectively halved the error rate of eigenface matching.

\section{Conclusion:-}

In this paper, we have displayed a broad audit of late research improvement on face recognition. Likewise this paper concentrated on face recognition frameworks, identification and restriction include extraction and recognition parts of the face recognition issue. Here we give underneath a brief rundown took after by conclusions in an indistinguishable request from the themes show up in the paper.

A significant stride in face recognition framework is the assessment and benchmarking of various calculations. A few imperative face databases and their related assessment techniques are checked on. The accessibility of these conventions which incorporate the FERET convention and the XM2VTS convention has significantly affected advance in the improvement of face recognition calculations.

\section{References:-}

1. Haiyuan Wu, QianChen, Masahiko Yachida, "Face Detection from Color Image usinga Fuzzy Pattern Matching Method", IEEE Transactions on Pattern Analysis and Machine Intelligence, Vol. 21, 1999, No. 6, pp. 557-563.

2. A S Georghiades, P N Belhumeur, D J Kriegman, "From Few too Many: Illumination Cone Models for Face Recognition under Variable Lighting and Pose", IEEE Transactions on Pattern Analysis and Machine Intelligence, Vol. 23, 2001, No. 6, pp. 643-660.

3. B Moghaddam, T Jebara, A Pentland, "Bayesian Face Recognition. Pattern Recognition", Vol. 33, 2000, No. 7, pp. 1771-1782.

4. K Nallaperumal, R Subban, K Krishnaveni, L Fred, R K Selvakumar, "Human Face Detection in Color Images using Skin Color and Template Matching Models for Multimedia on the Web", Wireless and Optical Communications Networks, 2006 IFIP International Conference on 11-13 April 2006, pp. 5-10

5. R Bruneli and T Poggio, "Face recognition: features versus templates", IEEE Transaction Pattern Analysis and Machine Intelligence, Vol. 15, 1993, pp. 1042-1052.

6. RJ Baron, "Mechanism of Human Facial Recognition", International Journal of Man Machine Studies, Vol. 15, 1981, pp. 137-178.

7. SushmaJaiswal, Sarita Singh Bhadauria, Rakesh Singh Jadon,"Comparison Between Face Recognition Algorithm-Eigenfaces, Fisherfaces And Elastic Bunch Graph Matching", Journal of Global Research in Computer Science, Vol. 2, No. 7, July 2011 
8. Raphael Feraud, Olivier J Bernier, Jean-Emmanuel Viallet and Michel Collobert,"A Fast and Accurate Face Detector Based on Neural Networks", IEEE Transactions on Pattern analysis and Machine Intelligence, Vol. 23, No. 1, 2001,pp. 42-53.

9. Henry A. Rowley, ShumeetBaluja and Takeo Kanade, "Neural Network-Based Face Detection", IEEE Transactions on Pattern analysis and Machine Intelligence, Vol. 20,No.1, 1998,pp. 23-30.

10. R A Fisher, "The Use of Multiple Measurements in Taxonomic Problems", Wiley Online Library, 1936.

11. PNBelhumeur, J PHespanda, DJKiregeman, "Eigenfaces vs. Fisherfaces: recognition using class Specific Linear Projection", IEEE Transaction on PAMI, Vol. 19, 1997,pp. 711-720

12. S Revathi, KRajakumar and G Deepa, "An Efficient Face Recognition System Using Curvelet With PCA", Asian Research Publishing Network, Vol.10, No.11,2015, pp.4915-4920.

13. FR Al-Osaimi, M Bennamoun and A Mian, "Integration of Local and Global Geometrical Cues for 3D Face Recognition," Pattern Recognition, Vol.41, 2008, pp.1030-1040.

14. B Gokberk, AA Salah and L Akarun, "Rank-Based Decision Fusion for 3D Shape-Based Face Recognition," IEEE 13th Signal Processing and Communications Applications Conference, 2005.

15. C L ShrinivasaNaika, Pradip K Das and Shivashankar B Nair, "Asymmetric Region Local Binary Pattern Operator for Person-dependent Facial Expression Recognition”, IEEE conference on Computing, Communication and Applications, 2012.

16. C Shan, S Gong and P W Mcowan, "Facial Expression Recognition Based On Local Binary Patterns: A Comprehensive Study", Image and Vision Computing, Vol. 27, no. 6, 2009, pp. 803- 816.

17. M Oravec, O Milos, P Jarmila, M Jan, O Lubos, F Matej and B Jozef, "Efficiency of Recognition Methods for Single Sample per Person Based Face Recognition", in Reviews, Refinements and New Ideas in Face Recognition, 2011

18. Ganapathi V Sagar, Savita Y Barker, K B Raja, K Suresh Babu, Venugopal K R “Convolution based Face Recognition using DWT and Feature Vector Compression", Third International Conference on Image Information Processing,2015.

19. Laurent El Shafey, ElieKhoury, and Sebastien' Marcel, “Audio-visual gender recognition in uncontrolled environment using variability modeling techniques", In International Joint Conference on Biometrics, 2014

20. HHan and A K Jain, "Age, Gender and Race Estimation From Unconstrained Face Images", MSU Technical Report MSU-CSE-14-5, Michigan State University, 2014.

21. S A Khan, M Ahmad, M Nazir, and N Riaz, "A Comparative Analysis Of Gender Classification Techniques", International Journal of Bio-Science and Bio-Technology, 2013,pp.223-244,.

22. Ravi J and K B Raja, "Hybrid Domain Based Face Recognition System", Internationa Journal of Advanced Networking and Applications, Vol. 03, Issue: 06, 2012, pp. 1402-1408. 